\title{
Modify Multistep Difference Transform Method for Solving Jamming Model in Lorentz System
}

\author{
Hartono $^{1, *}$ Agus M. Abadi ${ }^{1}$ Fitriana Y. Saptaningtyas ${ }^{1}$ \\ ${ }^{1}$ Department of Mathematics Education, Yogyakarta State University, Yogyakarta, Indonesia \\ *Corresponding author. Email: hartono@uny.ac.id
}

\begin{abstract}
The Lorentz system is one of the model which can be applied to single line traffic model. This model was based on the distance deviation, velocity deviation, and time acceleration. In this paper, we would like to solve the mathematical model of traffic congestion with the Lorentz system. Modify Multistep Difference Transform Method (MMDTM) is used to solve the model. Modification on initial value and several steps time interval is applied in some traffic congestion parameters. Convergence solutions was analysed and compared with the results when using the Runge Kutta method. The results show that both of the Modify Multi step DTM and Runge Kutta solutions are similar.
\end{abstract}

Keywords: Modify, Multistep, Difference Transform Method, Jamming Models, Lorentz System.

\section{INTRODUCTION}

Traffic congestions are problems that will occur continuously. Mathematical modeling can be used to investigate and predict traffic conditions. A model tree of the traffic model review has been proposed by [1]. Based on the used variables, the models could be categorized into three types: microscopic (trajectories, travel time, and distance variables), macroscopic (intensity, density and velocity variables) and mesoscopic (combined variables) [2]. Compared to the three models, microscopic models can preferable illustrate real traffic. $[3,4]$ have constructed microscopic models based on the Lorentz system. [5,6] have examined the local and global stability of the microscopic model of traffic congestion model with the Lorentz system.

Mathematical model of traffic congestion in the Lorentz system is a three dimensional nonlinear differential equation system. The transformation method using series is an alternative solution for its. [7] has used the Maclaurin series to conduct the convergence of Sech solution. Various methods for resolving approaches to solve the system of non-linear differential equations are Runge Kutta, variational iteration method, and DTM. [8] has examined the windup of initial value problems with DTM and related theorems. Accomplishment using series has advantages that do not need to be linearized, so that they are able to solve non-linear and high-order systems, [9]. Besides that, DTM has been able to solve the differential equation with the delay form [10]. At the solution of Difference Transform Method (DTM), usually it will generally experience divergence at long time intervals. This paper would like to discuss how to modify DTM so that it can converge throughout the time interval.

We would like to discuss the modeling of traffic congestion problems with the Lorentz system. The next section, we will discuss the solutions with DTM. Modification is made to the DTM so that it gets a convergent solution. The simulation results will be compared with the Runge Kutta method.

\section{RESULT}

This section is elaborated to explain the mathematical model's construction of traffic flow problem, which based on Lorentz model. Furthermore, the solution of the model was obtained by two methods: DTM method and modify DTM method. Ultimately, the Runge Kutta solution was computed to compare both solutions.

\subsection{Mathematical Modelling}

The microscopic model developed is a model based on how the vehicle maintains a safe distance. In this model, the vehicle's individual behavior can be observed. The variable in this model is the deviation between changes in position and the safe distance of the vehicle. Besides that, the deviation of the vehicle speed with the optimal speed of the vehicle, the last variable is the acceleration time which is the time needed by the vehicle to braking or reinforcing. The mathematical model was built with a Lorentz system which was developed by $[3,4,5,6]$. The Lorentz model of traffic congestion is a 
system of three-dimensional nonlinear differential equations as follow:

$$
\left\{\begin{array}{l}
\dot{\mu}=-\mu+v \\
\dot{v}=\frac{1}{\alpha}(\mu \tau-v) \\
\dot{\tau}=\frac{1}{\sigma}\left(\tau_{0}-\tau-\mu v\right)
\end{array}\right.
$$

With $\mu=$ distance deviation, $v=$ velocity deviation, $\tau$ $=$ acceleration time, $\alpha=\frac{t_{v}}{t_{\mu}}, \sigma=\frac{t_{\tau}}{t_{\mu}}$. The next section explains the existence of the equilibrium point of the traffic congestion model in Lorentz system.

\subsection{Solution Using DTM}

In this section, we will explain how to find a solution for a non-linear differential equation system using DTM. Previously, the DTM transformation function will be used which is requisite to find congestion model solutions in the Lorentz system.

Given transformation using DTM:

$$
Y(k)=\left.\frac{1}{k !}\left(\frac{d^{k}}{d x^{k}} y(x)\right)\right|_{x=x_{0}}
$$

Which has an inverse function that is

$$
y(x)=\sum_{k=0}^{\infty} Y(k)\left(x-x_{0}\right)^{k}
$$

Inverse functions are the solutions of a non-linear differential equations system. As for the related theorems are:

Theorem 1. If $y(x)=y_{1}(x) \pm y_{2}(x)$, then $Y(x)=Y_{1}(x) \pm Y_{2}(x)$.

Theorem 2. If $y(x)=c$ for $c$ is real number, then $Y(k)=\delta(k)=\left\{\begin{array}{l}1, \text { for } k=0 \\ 0, \text { for } k \neq 0\end{array}\right.$.

Theorem 3. If $y(x)=\frac{d^{n} y_{1}(x)}{d x^{n}}$, then $Y(k)=\frac{(k+n) !}{k !} Y_{1}(k+n)$.

Theorem 4. If $y(x)=y_{1}(x) y_{2}(x)$, then $Y(k)=\sum_{k_{1}=0}^{k} Y_{1}\left(k_{1}\right) Y_{2}\left(k-k_{1}\right)$.

To find the solutions of the model, the first equation in System 1 is transformed using the Theorem 1 and Theorem 3, so that it is obtained

$$
\tilde{\mu}(k+1)=\frac{-\tilde{\mu}(k)+\tilde{v}(k)}{k+1}
$$

The second equation in System 1 is transformed using the Theorems 1,3, and 4, so that they are obtained:

$$
\tilde{v}(k+1)=\frac{\sum_{k_{1}=0}^{k} \tilde{\mu}\left(k_{1}\right) \tilde{\tau}\left(k-k_{1}\right)-\tilde{v}(k)}{\alpha \cdot(k+1)}
$$

The third equation in System 1 is transformed using Theorems 1,2,3, and 4, so that it is obtained:

$$
\tilde{\tau}(k+1)=\frac{\tau_{0}-\tilde{\tau}(k)-\sum_{k_{1}=0}^{k} \tilde{\mu}\left(k_{1}\right) \tilde{v}\left(k-k_{1}\right)}{\sigma \cdot(k+1)}
$$

After transformation, inverse transformation is done to find the solution. As for the detailed steps in the settlement using DTM are described.

The process of the DTM can be described as:

1. Apply the differential transform to initial condition for every initial value in each variable.

2. Apply the differential transform to the differential equation system to obtain a recursion equation. Theorems 1, 2, 3, and 4 are used in govern the solutions of jamming model using Lorentz system.

3. Use the transformed initial condition on the first step and the recursion equation on the second step to determine the unknowns $Y_{0}, Y_{1}, Y_{2}, \ldots$.

4. Use the differential inverse transform formula to obtain an approximate solution for initial-value problem

\subsection{Solution using Modify DTM Multistep}

The process of the Modify DTM multistep can be described as:

1. Divide the time interval domain, provided that the serial solution will converge according to the number of iterations performed. The smaller the time interval and the more iterations, the cutting error will decrease. At this paper, the time interval 1 and 5 iteration are selected.

2. Apply the differential transform to initial condition for every initial value for each variable at the first time interval.

3. Apply the differential transform to the differential equation system to obtain a recursion equation at the first time interval. The theorem 1,2,3, and 4 are used to transform congestion model in Lorentz system.

4. Use the transformed initial condition on first step and the recursion equation on second step to determine the unknowns $Y_{0}, Y_{1}, Y_{2}, \ldots$ in first time interval step.

5. Use the differential inverse transform formula to obtain an approximate solution for initial-value problem in first time interval step.

6. Update the initial value of the settlement after being transformed inversely at the end of the interval. Do the process again at step two and so on. Select the 
desired iteration, may differ from the first time interval.

7. Repeat up to all specified time intervals.

\section{SIMULATION OF JAMMING MODEL USING MODIFY MULTISTEP DTM}

In this paper some simulations will be explained in each possible parameter combination. Parameters that affect the stability of the Lorentz system based on [5,6] are $\tau_{0}$. In this simulation, it will be adjusted to the possible value of $\tau_{0}$, which are less than one, equal to one, and more than one.

Case 1: Solution of Jamming model in Lorentz system with some parameter and $\tau_{0}<1$
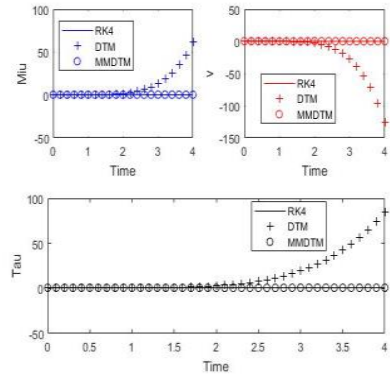

Fig. 1. A. $\alpha=0.6, \sigma=0.8$
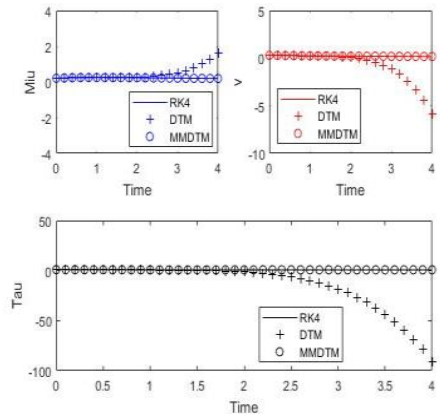

Fig. 1. C. $\alpha=1 \cdot 6, \sigma=0.4$
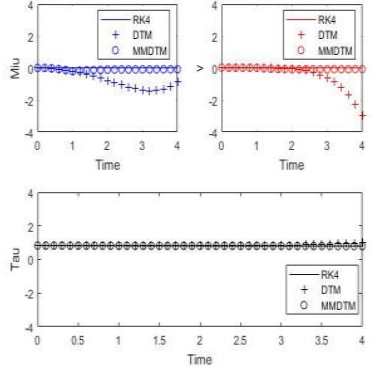

Fig. 1. B. $\alpha=0.8, \sigma=1.5$

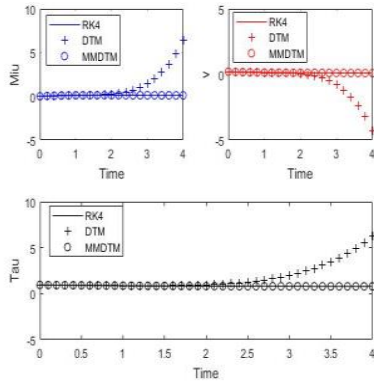

Fig. 1. D. $\alpha=1.7, \sigma=1.4$

Figure 1. A, B, C, and D show if $\tau_{0}<1$ and all of the possible $\alpha, \sigma$ then the DTM converges until $t=1$. After that, the solution will be divergent. Contradiction with the MMDTM i.e the settlement will continue to converge for the entire time interval. Updating the initial value construct new series which is more reliable. This shows that the solution of this modification method is similar to the Runge Kutta method. Furthermore, the accomplishment behavior will go to equilibrium $\left(0,0, \tau_{0}\right)$ in line with the results $[5,6]$.
Case 2: Solution of Jamming model in Lorentz system with some parameter and $\tau_{0}=1$

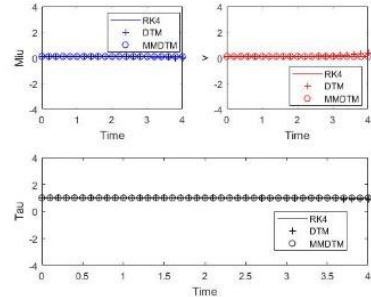

Fig. 2. A. $\alpha=0.6, \sigma=0.8$
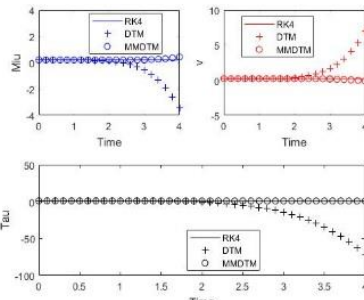

Fig. 2. C. $\alpha=1.6, \sigma=0.4$.

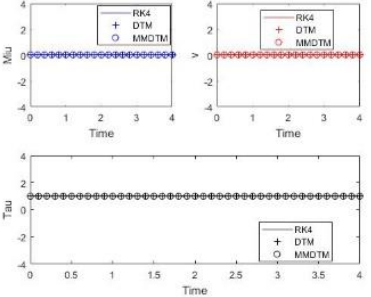

Fig. 2. B. $\alpha=0.8, \sigma=1.5$
Figure 2. A, B, C, and D indicates if $\tau_{0}=1$ and all of the possible $\alpha, \sigma$ then the DTM converges until $t=1$. After that, the solution will be not converge. Contrariety with the DTM modification i.e the completions will keep on to converge for the whole time interval. This indicates that the solution of this modification method is conformable to the Runge Kutta method. Updating the initial value in each step govern new series which is more accurate. Furthermore, the accomplishment behavior will go to equilibrium $\left(0,0, \tau_{0}\right)$ in line with the results $[5,6]$.

Case 3: Solution of Jamming model in Lorentz system with some parameter and $\tau_{0}>1$

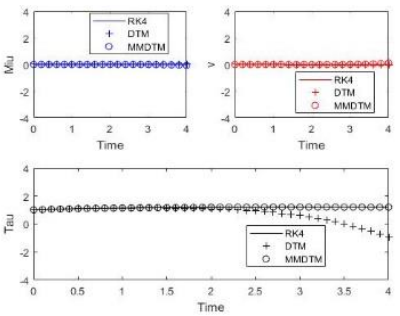

Fig. 3. A. $\alpha=0.6, \sigma=0.8$
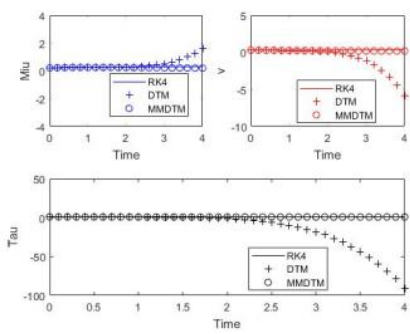

Fig. 3. C. $\alpha=1.6, \sigma=0.4$.

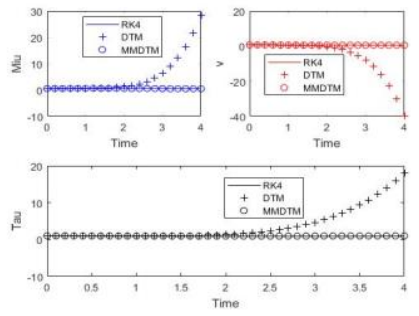

Fig. 3. B. $\alpha=0.8, \sigma=1.5$

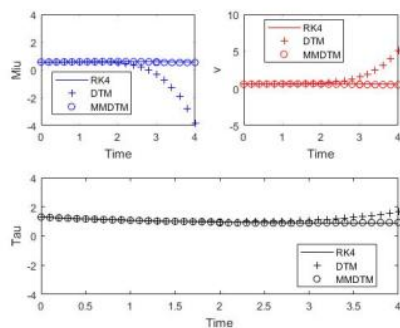

Fig. 3. D. $\alpha=1.7, \sigma=1.4$ 
Figure 3. A, B, C, and D represent if $\tau_{0}>1$ and all of the possible $\alpha, \sigma$ then the DTM converges until $t=1$. After that, the solution will be divergent. The distinction with the DTM modification, the solutions will continue to converge for the time interval given. This represents that the solution of this modification method is tantamount to the Runge Kutta method. Renewing the initial value in each step establish new solutions which are more precise. Furthermore, the accomplishment behavior will go to equilibrium $\left(\sqrt{\tau_{0}-1}, \sqrt{\tau_{0}-1}, 1\right)$. It accords with the results $[5,6]$.

\section{CONCLUSION}

The results showed that the solutions of the jamming model in Lorentz system using two methods gave the similar results. Both methods are Modify DTM Multi step and Runge Kutta. Modifications are made to the construction of transformations at each time interval by changing the initial value and being repeated over all specified time intervals. From the simulation results it can be seen that in all possible traffic congestion parameters, the DTM modification gives good results. The results indicate that the solutions are in accordance with the reality.

\section{REFERENCES}

[1] Van Wageningen-Kessels F, Van Lint H, Vuik K and S Hoogendoom . EURO J Transp Logist. Vol 4,pp. 445. 2015.

[2] R Mardiati, N Ismail and A. Faroqi . ARPN Journal of Engineering and Applied Sciences. vol 9, pp. 1794. 2014.

[3] Khomenko D and Yuschenko O. Ser of Pschyc Journal. vol 37 pp. 44. 2013.

[4] Olemskoi A I, Khomenko A V. Journal of Phys Rev. Vol 63 pp. 0361161. 2001.

[5] Hartono, F Y Saptaningtyas and K P Krisnawan. J. Phys.: Conf. Ser. vol 983 pp. 012092. 2018.

[6] Hartono, A M Abadi, F Y Saptaningtyas and N Binatari. Far East J. Math. Sci. vol 98 , pp 245. 2018.

[7] U. Al Khawaja and Qasem M. Al-Mdallal. International Journal of Differential Equations. Vol 2018. 2018.

[8] A. S. Oke. Journal of Advances in Mathematics and Computer Science. vol 24 pp. 6. 2017.

[9] Brahim Benhammouda . SpringerPlus. vol 4 , pp.648. 2015 .

[10] Benhammouda and Vazquez-Leal. SpringerPlus. vol, 5, pp. 1723. 2016. 